\title{
Poli(4-vinilbenzil-g-stiren) Aşı Kopolimerinin RAFT ve SRP Yöntemleriyle Sentezi ve Karakterizasyonu
}

\author{
Bedrettin SAVAŞ ${ }^{1 *}$, Temel ÖZTÜRK ${ }^{2}$, Ergül MEYVACI ${ }^{2}$ \\ ${ }^{1}$ Kafkas Üniversitesi, Kars Meslek Yüksekokulu, 36100 Kars, Türkiye \\ ${ }^{2}$ Giresun Üniversitesi, Kimya Bölümü, 28200 Giresun, Türkiye
}

(ORCID: 0000-0002-6620-5493) (ORCID: 0000-0002-7856-9809) (ORCID: 0000-0003-3530-1316)

\begin{abstract}
$\ddot{O} \mathbf{z}$
Poli(4-vinilbenzil-g-stiren) [poli(VB-g-S)] aşı kopolimeri tersinir katılmalı/ayrışmalı zincir transfer (RAFT) ve serbest radikal polimerizasyon (SRP) teknikleri kullanılarak sentez edildi. Bu amaç için, 4-vinilbenzil klorür monomeri kullanılarak SRP yöntemiyle poli(4-vinilbenzil klorür) [poli-4-VBC] polimeri sentezlendi. İkinci aşamada, poli-4-VBC polimeri ile potasyum etil ksantat reaksiyona sokularak makro-RAFT ajanı oluşturuldu. Son aşamada, RAFT tekniğiyle makro-RAFT ajanı varlığında stiren monomeriyle poli(VB-g-S) aşı kopolimeri sentez edildi. Sentezlenen ürünler ${ }^{1}$ H-NMR, FT-IR, TGA, SEM ve Dinamik Işık Saçılımı (DLS) cihazları gibi spektroskopik ve termal analiz teknikleri kullanılarak karakterize edildi.
\end{abstract}

Anahtar kelimeler: Aş1 kopolimer, 4-vinilbenzil klorür, stiren, makro-RAFT ajanı, serbest radikal polimerizasyonu, tersinir katılmalı/ayrışmalı zincir transfer polimerizasyonu.

\section{Synthesis and Characterization of Poly(4-vinylbenzyl-g-styren) Graft Copolymer by RAFT and FRP Methods}

\begin{abstract}
Synthesis of poly(4-vinylbenzyl-g-styrene) [poly(VB-g-S)] graft copolymer was carried out by free radical polymerization (FRP) and reversible addition-fragmentation chain transfer polymerization (RAFT) methods. For this purpose, poly(4-vinylbenzyl chloride) [poly-4-VBC] was synthesized using 4-vinylbenzyl chloride by FRP. In second stage, macro-RAFT agent was synthesized by chemical reaction of poly-4-VBC with potassium ethyl xanthogenate. In last stage, macro-RAFT agent and styrene were used to obtain poly(VB-g-S) graft copolymer by RAFT polymerization. Product characterization was fulfilled by using spectroscopic and thermal analysis techniques such as ${ }^{1} \mathrm{H}-\mathrm{NMR}$, FT-IR, TGA, SEM and Dynamic Light Scattering (DLS) instruments.
\end{abstract}

Keywords: Graft copolymer, 4-vinylbenzyl chloride, styrene, macro-RAFT agent, free radical polymerization, reversible addition-fragmentation chain transfer polymerization.

\section{Giriş}

Son yıllarda, sadece yeni tür plastik malzemelerin sentezlenmesi değil, aynı zamanda yeni uygulamaların getirdiği gereklilikleri karşılamak için var olan polimerlerin modifiye edilmesi ayrı bir önem kazanmaktadır. Aşı kopolimerizasyon makro molekül özelliklerini geliştirme yöntemlerinden biridir [1]. Farklı kimyasal ya/ya da fiziksel özelliklere sahip iki ayrı monomerin değişen oranlarda aynı makro moleküle dâhil edilmesi ticari ve bilimsel önem arz eden yeni malzemelerin meydana gelmesine katkı sağlamaktadır [2-8]. Kopolimerlerin hazırlanmasında bazı dezavantajlara sahip farklı organik reaksiyonlar kullanılmaktadır. Bu dezavantajların üstesinden gelebilmek için, kontrollü (yaşayan) serbest radikal polimerizasyon tekniklerinin araştırılması son yıllarda önemli bir şekilde artmıştır. Bir radikal polimerizasyon tekniği olan serbest radikal polimerizasyonu (SRP) çok farklı sayıda polimer elde etmek için önemli sentez tekniklerinden biridir $[9,10]$. Ayrıca SRP, endüstriyel polimer üretimi

*Sorumlu yazar: bdrttn.svs.36@gmail.com

Geliş Tarihi: 01.06.2021, Kabul Tarihi: 01.09.2021 
için en önemli süreçlerden biridir. Çünkü daha geniş bir monomer sınıfıyla daha kolay deneysel koşullar altında polimerizasyon gerçekleştirilebilir [11-14]. Son zamanlarda kontrollü polimerizasyon proseslerinden başlayarak polimer zincir sonu fonksiyonelliğine sahip ya da aş1 zincir kısmının oluşturulduğu kompleks mimarili makro moleküller sıklıkla sentezlenmektedir. Bu makro moleküllerin sentezi için tersinir katılmalı/ayrışmalı zincir transfer (RAFT) polimerizasyonu en son geliştirilen kontrollü radikal polimerizasyon yöntemini temsil eder ve çok iyi tanımlanmış çeşitli polimerlerin sentezi için güçlü bir tekniktir [15]. Kontrollü radikal polimerizasyonların genel avantajları olan polimer mimarisinin ve molekül ağırlığının önceden ayarlanabilmesi ile düşük molekül ağırlığı dağılımına (dispersiteye) sahip polimerlerin üretimi RAFT'ın da önemli avantajları arasındadır [16-26]. Termoplastik özellik gösteren polistiren, birçok metale göre üretiminin oldukça ucuz ve diğer malzemelere iyi bir alternatif olması, sahip olduğu özelliklerin geliştirilerek yeni özellikler kazandırılması ihtiyacını gerektirmiştir [27].

Bu çalışmada; SRP ve RAFT teknikleri kullanılarak poli(4-vinil benzil-g-stiren) [poli(VB-g-S)] aşı kopolimerleri sentez edildi. Aşı kopolimer, spektroskopik yöntemler olan FT-IR, ${ }^{1} \mathrm{H}-\mathrm{NMR}$, DLS ve SEM analizleri ile karakterize edildi. Aşı kopolimerin termal geçişleri ve bozulma özellikleri TGA yöntemi kullanılarak incelendi.

\section{Materyal ve Metot}

\subsection{Kullanılan kimyasallar}

2,2'-azobisizobutironitril (AIBN), Aldrich ürünü olup alındığı gibi tepkimelerde kullanıldı. Potasyum etil ksantat, $N, N$-dimetilformamit (DMF) ve metanol Sigma-Aldrich firmasindan temin edildi. 4-vinil benzil klorür ve toluen Merck ürünü olup alındığg gibi kullanıldı. Stiren, Merck ürünü olup inhibitörü uzaklaştırmak için alümina kolonundan geçirildi. Tetrahidrofuran (THF) Honeywell, dietil eter ve petrol eteri Riedel-de Haen firmalarından temin edildi.

\subsection{Kullanılan cihazlar}

${ }^{1}$ H-NMR spektrumları, 400 MHz'lik Bruker Ultra Shield Plus cihazı kullanılarak kaydedildi. FT-IR spektrumları, 400-4000 $\mathrm{cm}^{-1}$ aralığında Alpha-P Bruker FT-IR spektrometresi kullanılarak elde edildi. Dinamik Işık Saçı1ımı (DLS) cihazı olan Insmark IR120 model refraktometre ile Malvern Zetasizer Nano Serisi Nano-ZS kullanarak homo polimerin ve aşı kopolimerin ağırlıkça ortalama molekül ağırlıkları bulundu (çözücü: DMF; konsantrasyon: $1 \mathrm{mg} / \mathrm{mL}, 0.5 \mathrm{mg} / \mathrm{mL}$, ve $0.25 \mathrm{mg} / \mathrm{mL}$; sıcaklık: 20 ${ }^{\circ} \mathrm{C} ; \lambda=633 \mathrm{~nm}$ ). Carl Zeiss Sigma 300 Field taramalı elektron mikroskobuyla (SEM) aşı kopolimerin üç boyutlu (3D) görüntülerinin alınmasında kullanıldı. Termogravimetrik analiz (TGA) ölçümü Seiko II Exstar 6000 model alet kullanarak yapıldı. Örnek, $25^{\circ} \mathrm{C}$ 'den $600{ }^{\circ} \mathrm{C}$ ye $10^{\circ} \mathrm{C} /$ dakika hızla azot gazı atmosferi altında 1sitild1.

\subsection{Serbest radikal polimerizasyonu (SRP) yöntemiyle poli(4-vinilbenzil klorür) [poli-4-VBC] polimerin sentezi}

$25 \mathrm{~mL}$ 'lik bir cam balona çözücü olarak $3 \mathrm{~mL}$ toluen konulduktan sonra sirasıyla monomer olarak $2.0357 \mathrm{~g}$ 4-vinil benzil klorür ve başlatıcı olarak da $0.0140 \mathrm{~g}$ AIBN ilave edildi. İnert ortam sağlamak amacıyla balon içeriği yaklaşık 5 dakika boyunca argon gazından geçirilerek balonun ağzı kapatıldı. Bir 1sıtıcılı manyetik karıştırıcı üzerinde termal olarak $90^{\circ} \mathrm{C}$ 'ye ayarlanmış yağ banyosu içerisine balon daldırılarak 24 saat sürede karıştırıldı. Bu süre sonunda balon içeriği soğutularak aşırı metanolde çöktürüldü. Dekante işleminden sonra vakum etüvünde $40{ }^{\circ} \mathrm{C}^{\prime}$ de 24 saat kurutuldu. Sentezlenen poli4-VBC polimeri tartılarak verim hesapland. 


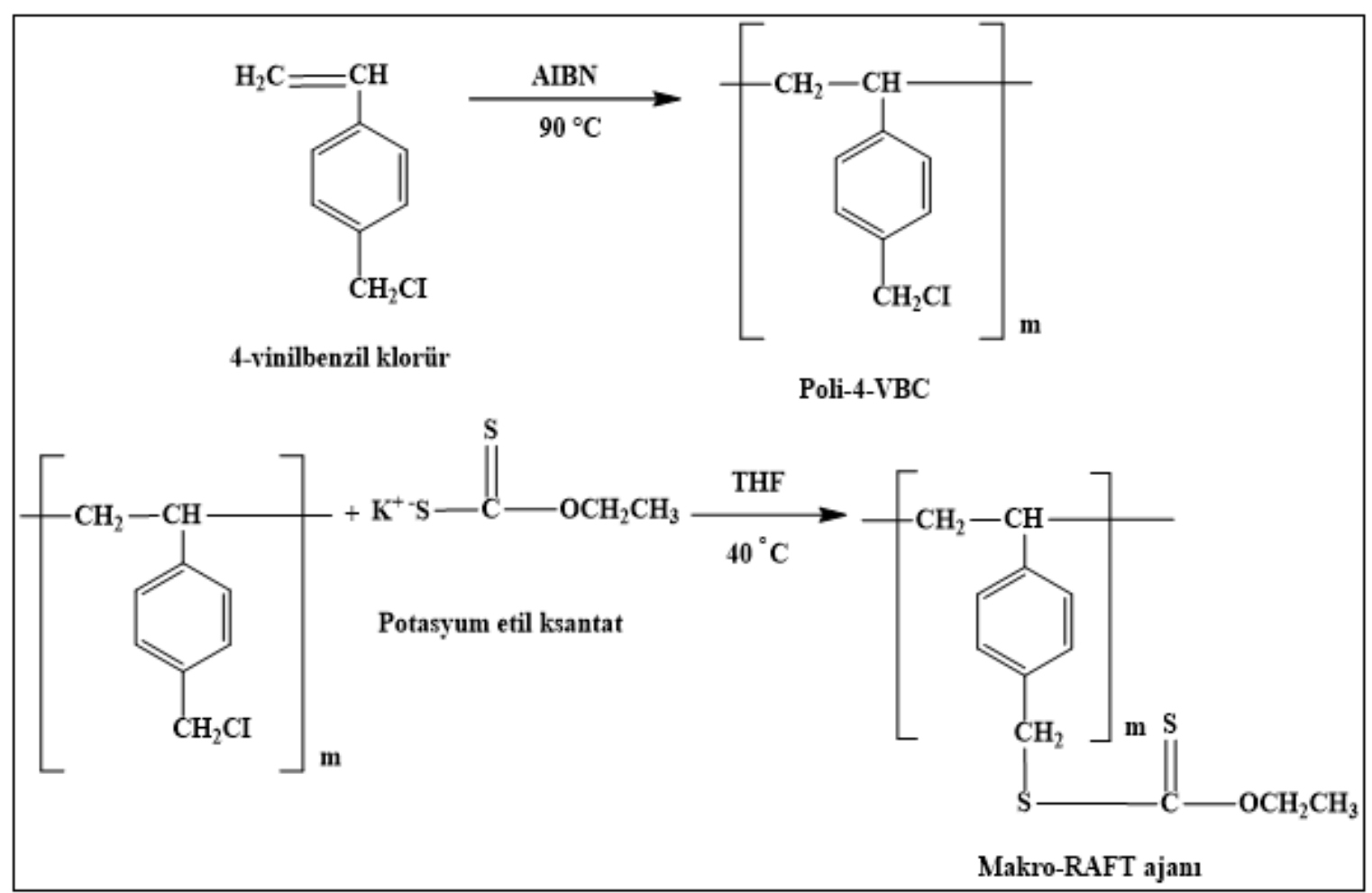

Şekil 1. Poli-4-VBC homo polimerinin ve makro-RAFT ajanının sentez mekanizması

\subsection{Potasyum etil ksantat kullanılarak makro-RAFT ajanının sentezi}

Bir deney tüpü içerisine $0.1520 \mathrm{~g}$ poli-4-VBC homo polimeri $3 \mathrm{~mL}$ THF çözücüsünde çözerek 0.3018 g potasyum etil ksantat ilave edildi. Tüp argon gazıyla degaz edilerek $40^{\circ} \mathrm{C}$ 'de 72 saat karıştırıldı. Tüp muhteviyatı süzgeç kâğıdıyla süzüldükten (reaksiyona girmeyen potasyum etil ksantat uzaklaştırılması için) sonra dietil eter/petrol eteri (hacimce 1/1) karışımı kullanılarak çöktürüldü. Dekande işleminden sonra ürün oda sıcaklığında vakum etüvünde kurutuldu ve tartıldı. Yüzde verim hesaplandı.

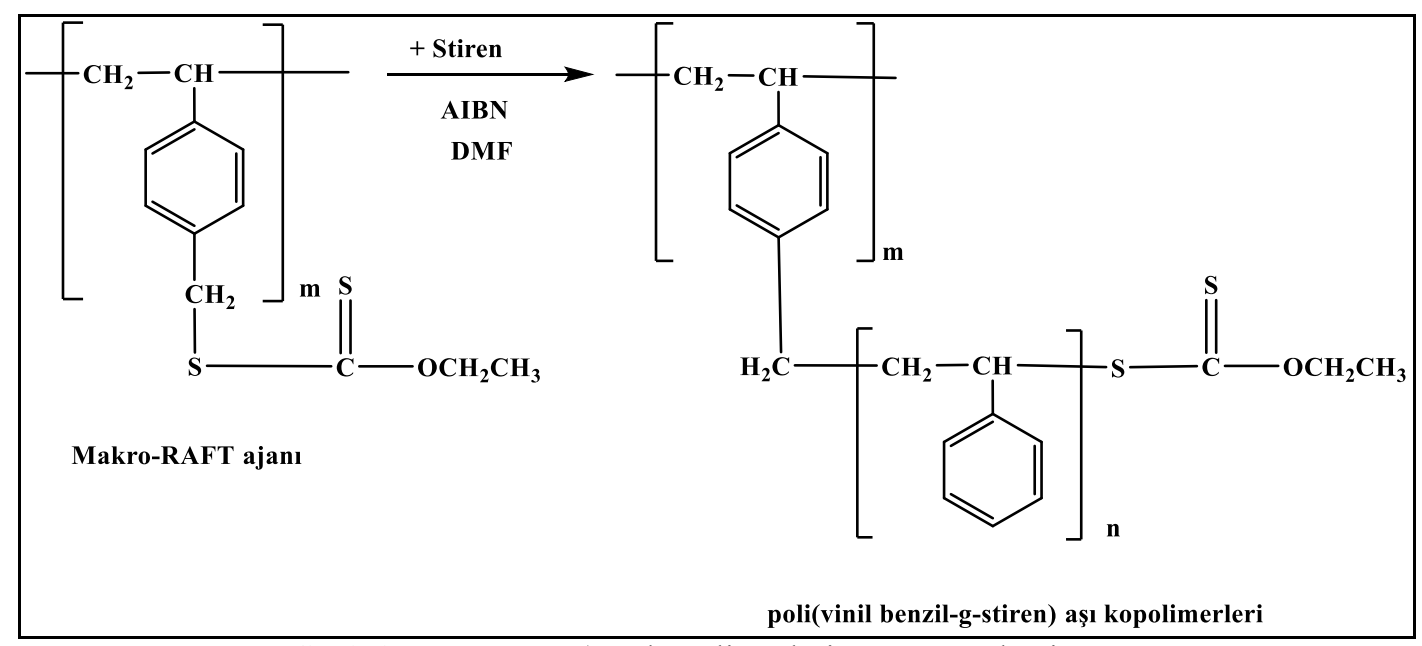

Şekil 2. Poli(VB-g-S)aşı kopolimerlerin sentez mekanizması

\subsection{RAFT polimerizasyonuyla poli(VB-g-S) aşı kopolimerlerin sentezi}

Bir reaksiyon balonuna sirasıyla $0.0227 \mathrm{~g}$ makro-RAFT ajanı, 1,0045 g stiren monomeri, eser miktarda AIBN ve $3 \mathrm{~mL}$ THF eklendi ve karışım $5 \mathrm{dk}$ boyunca argon gazından geçirilerek $90{ }^{\circ} \mathrm{C}$ 'de 20 saat karıştırıldı. Daha sonra metanolde çöktürüldü. Dekante işleminden sonra vakum etüvünde kurutularak tartıld. Verim hesabı yapıldı. 


\section{Bulgular ve Tartışma}

\subsection{Poli-4-VBC polimerin sentezi}

Poli-4-VBC homo polimerin sentez mekanizması Şekil 1'de verilmiştir. Poli-4-VBC homo polimeri ağırlıkça \% 60,32 verimle elde edildi. Şekil 3' de poli-4-VBC homo polimerin FT-IR spektrumuna göre; $3060-3025 \mathrm{~cm}^{-1}$ aromatik -CH grubunun, $2920-2850 \mathrm{~cm}^{-1}$ alifatik $-\mathrm{CH},-\mathrm{CH}_{2}$ grubunun, $1420 \mathrm{~cm}^{-1}$ aromatik $-\mathrm{C}=\mathrm{C}$ grubunun ve $821 \mathrm{~cm}^{-1}-\mathrm{C}-\mathrm{CI}$ grubunun pikleri görülmektedir. Şekil $4 \mathrm{a}$ 'da gösterilen poli4-VBC homo polimerin ${ }^{1} \mathrm{H}-\mathrm{NMR}$ spektrumuna göre; $(\delta, \mathrm{ppm})$ : 7.1-6.5 (c, aromatik $\left.\mathrm{CH}\right), 4.7\left(\mathrm{~d}, \mathrm{CH}_{2} \mathrm{CI}\right)$, $1.7(\mathrm{~b}, \mathrm{CH})$ ve $1.5\left(\mathrm{a}, \mathrm{CH}_{2}\right)$ protonlarının pikleri görülmektedir. Dinamik Işık Saçılımı (DLS) cihazı kullanılarak homo polimerin ağırlıkça ortalama molekül ağırlığı (Mw) 57200 Da olarak bulundu (Şekil 7a).

\subsection{Makro-RAFT ajanının sentezi}

Makro-RAFT ajanının sentez mekanizması Şekil 1'deki ikinci satırda verilmiştir. Makro-RAFT ajanı ağırlıkça \% 86,78 verimle elde edildi. Şekil 3'de makro-RAFT ajanının FT-IR spektrumuna göre; 3065 $3020 \mathrm{~cm}^{-1}$ aromatik $-\mathrm{CH}$ grubunun, $2980-2870 \mathrm{~cm}^{-1}$ alifatik $-\mathrm{CH},-\mathrm{CH}_{2}$ grubunun, $1444 \mathrm{~cm}^{-1}$ aromatik $\mathrm{C}=\mathrm{C}$ grubunun ve $1041 \mathrm{~cm}^{-1}-\mathrm{SC}$ grubunun pikleri görülmektedir.

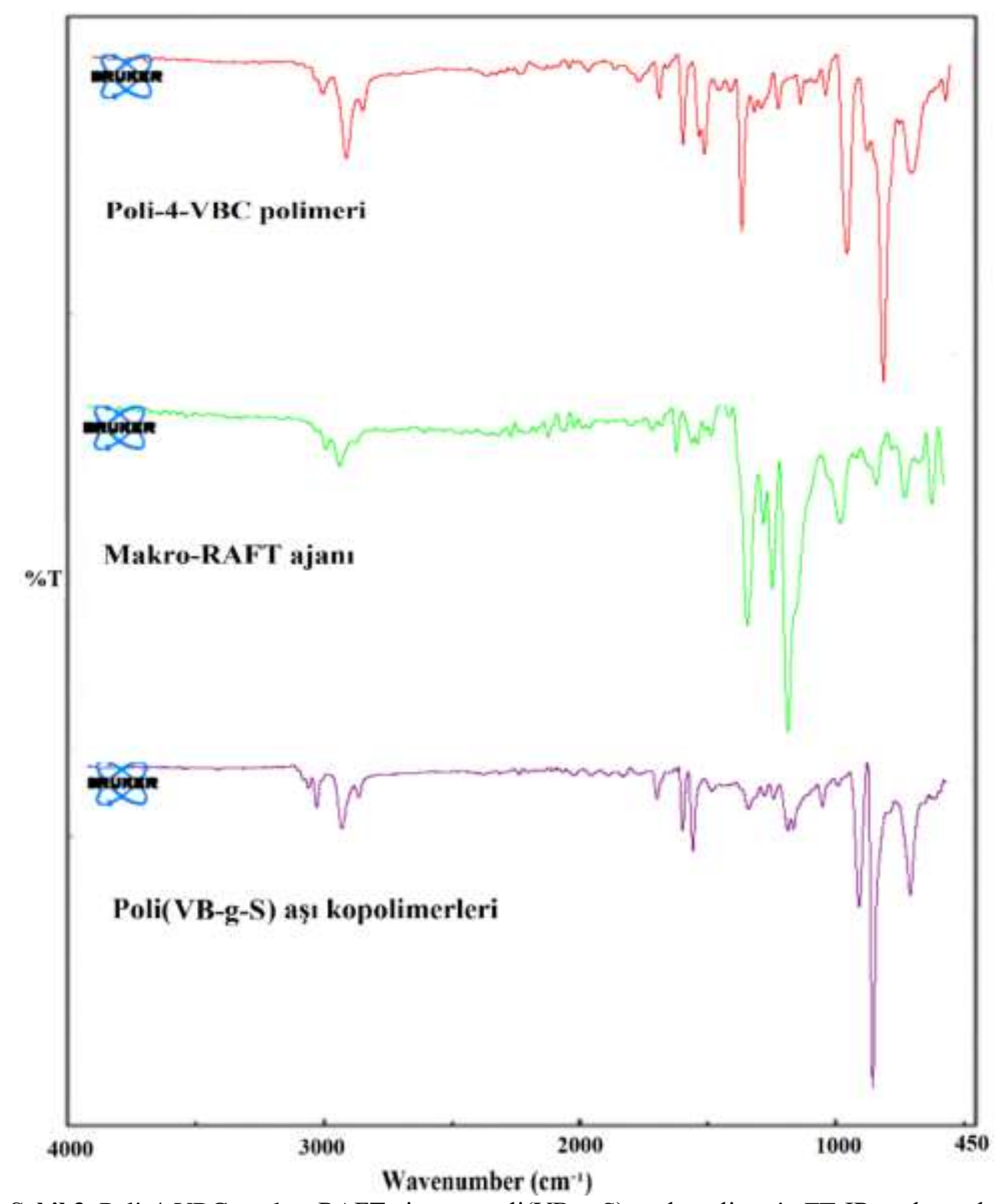

Şekil 3. Poli-4-VBC, makro-RAFT ajanı ve poli(VB-g-S) aşı kopolimerin FT-IR spektrumları 


\subsection{Poli(VB-g-S) aşı kopolimerlerin sentezi}

Poli(VB-g-S) aşı kopolimerin sentez mekanizması Şekil 2'de verilmiştir. Aşı kopolimeri ağırlıç̧a \% 84,42 verimle elde edildi. Şekil 3'deki poli(VB-g-S) aşı kopolimerin FT-IR spektrumuna göre; 3058$3024 \mathrm{~cm}^{-1}$ aromatik $-\mathrm{CH}$ grubunun, 2918-2848 $\mathrm{cm}^{-1}$ alifatik $-\mathrm{CH},-\mathrm{CH}_{2}$ grubunun, $1447 \mathrm{~cm}^{-1}$ aromatik $\mathrm{C}=\mathrm{C}$ grubunun ve $1050 \mathrm{~cm}^{-1}$-SC grubunun pikleri görülmektedir. Şekil $4 \mathrm{~b}$ 'de gösterilen poli(VB-g-S) aşı kopolimerin ${ }^{1} \mathrm{H}-\mathrm{NMR}$ spektrumuna göre; $(\delta, \mathrm{ppm}):$ 7.1-6.5 (c,g, aromatik $\left.\mathrm{CH}\right), 3.3\left(\mathrm{~h}, \mathrm{CH}_{2} \mathrm{O}\right), 1.8$ (b,f CH) ve $1.3\left(\mathrm{a}, \mathrm{e} \mathrm{CH}_{2}\right) 1.0\left(1, \mathrm{CH}_{3}\right)$ protonlarının pikleri görülmektedir. Dinamik Işık Saçılımı (DLS) cihazı kullanılarak aşı kopolimerin ağırlıkça ortalama molekül ağırlı̆ı (Mw) 146000 Da olarak bulundu (Şekil 7b). Poli(VB-g-S) aşı kopolimerin molekül ağırlığının poli-4-VBC homo polimerinkinden daha büyük olması kopolimerin başarılı bir şekilde sentezlendiğinin diğer bir kanıtıdır.

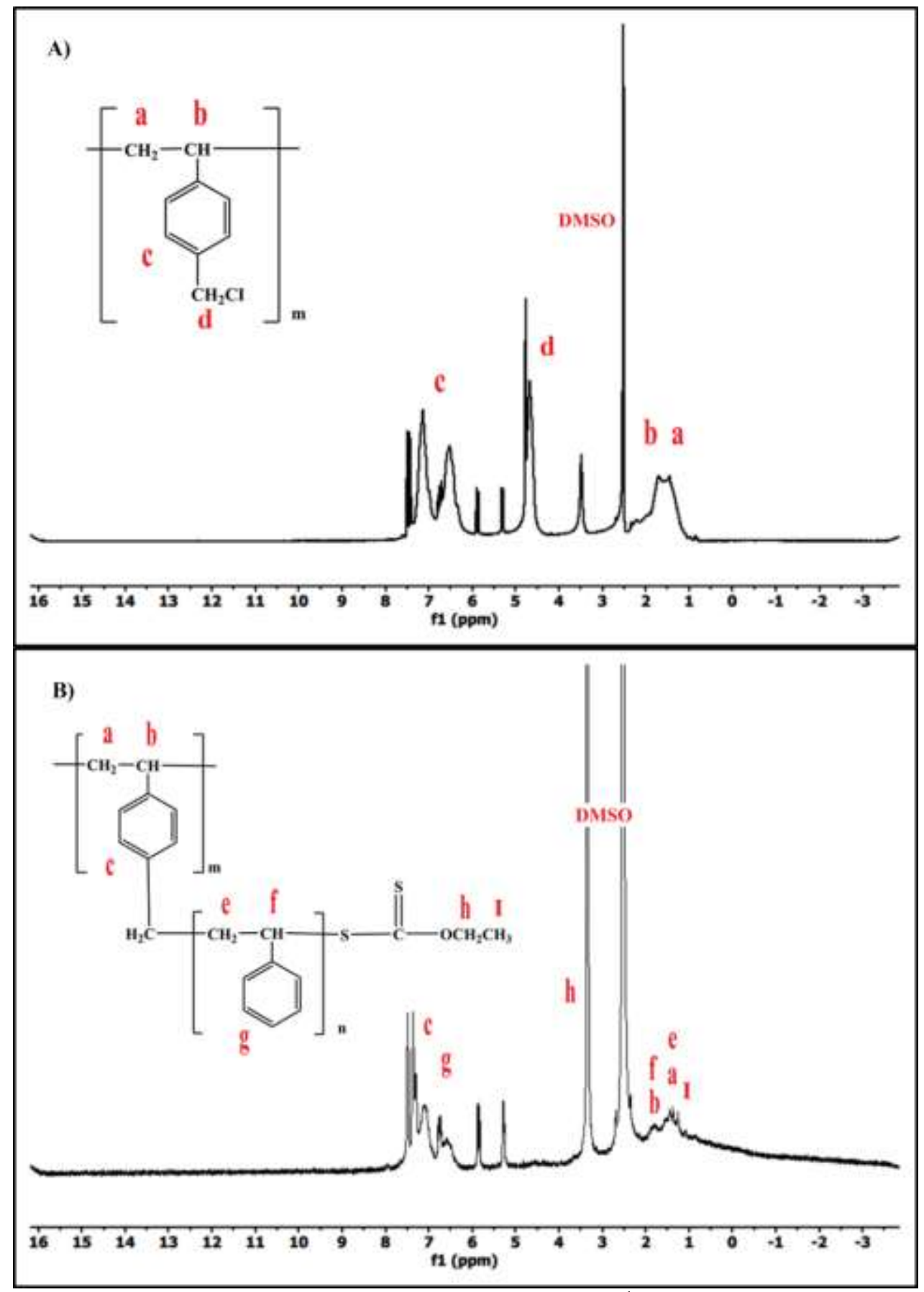

Şekil 4. Poli-4-VBC ve poli(VB-g-S) aşı kopolimerin ${ }^{1} \mathrm{H}-\mathrm{NMR}$ spektrumları 
Poli(VB-g-S) aş1 kopolimerin yüzey morfolojisi incelemek için SEM görüntüleri alınmıştır (Şekil 5). Polimer örneği ince bir altın tabaka ile kaplandı. Polimer örneğinin yüzey fotoğrafları farklı büyültme boyutlarında alındı. SEM görüntülerinde gözenekli yapılar görülmektedir. Aş1 kopolimerinin SEM görüntüleri pürüzlü bir yüzey gösteren sürekli bir faz oluşturmuş.
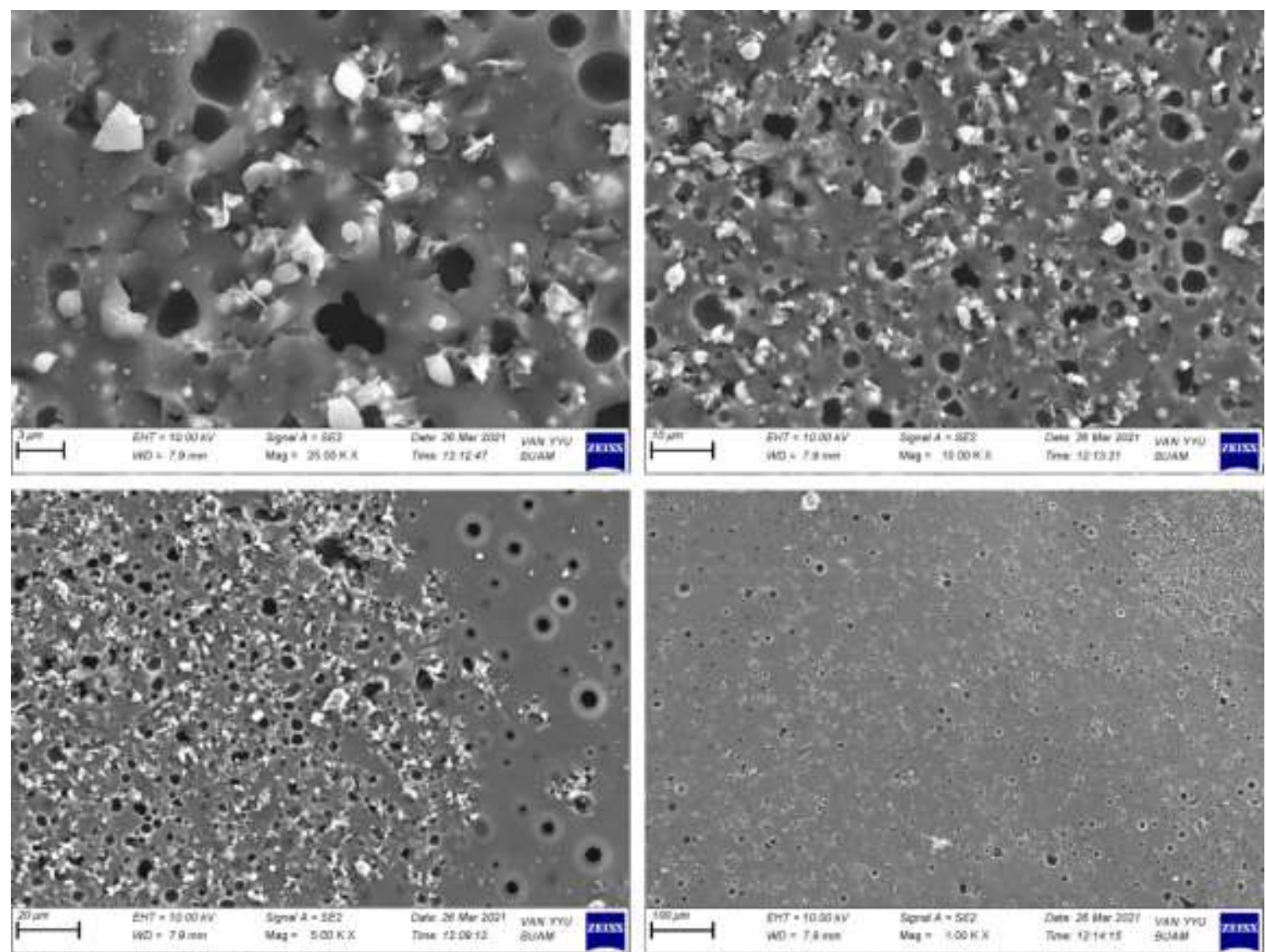

Şekil 5. Poli(VB-g-S) aşı kopolimerin SEM görüntüleri (X25000, X10000, X5000, X1000)

Poli(VB-g-S) așı kopolimerin TGA eğrisine bakıldığında yaklașık $300{ }^{\circ} \mathrm{C}$ 'den başlayıp 480 ${ }^{\circ} \mathrm{C}$ 'ye kadar bozunmanın gerçekleştiği; $\mathrm{Td}_{1}=401{ }^{\circ} \mathrm{C}$ ve $\mathrm{Td}_{2}=418{ }^{\circ} \mathrm{C}$ olmak üzere iki ayrı bozunma sıcaklığında bozunmanın maksimum olduğu görülmektedir. Reaksiyonlarda kullanılan monomerlerin yapısal benzerliklerinden dolayı Td'lerin birbirine yakın olduğu görülmüştür (Şekil 6).

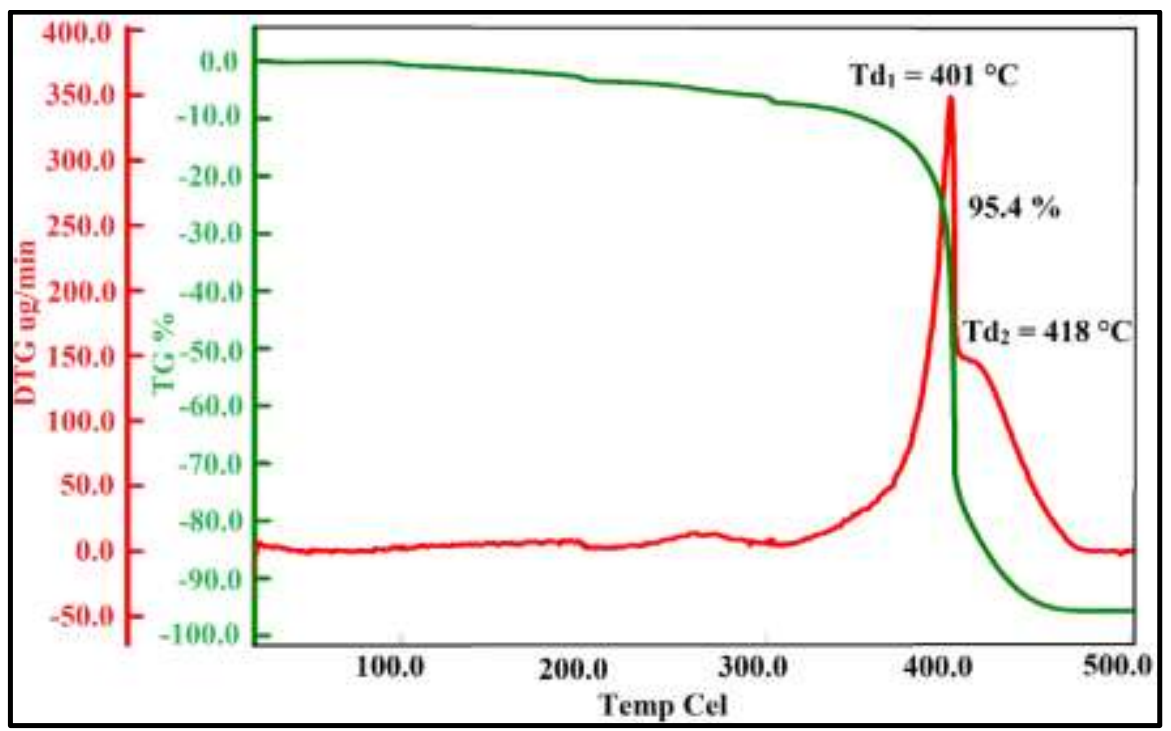

Şekil 6. Poli(VB-g-S) aşı kopolimerin TGA eğrisi 


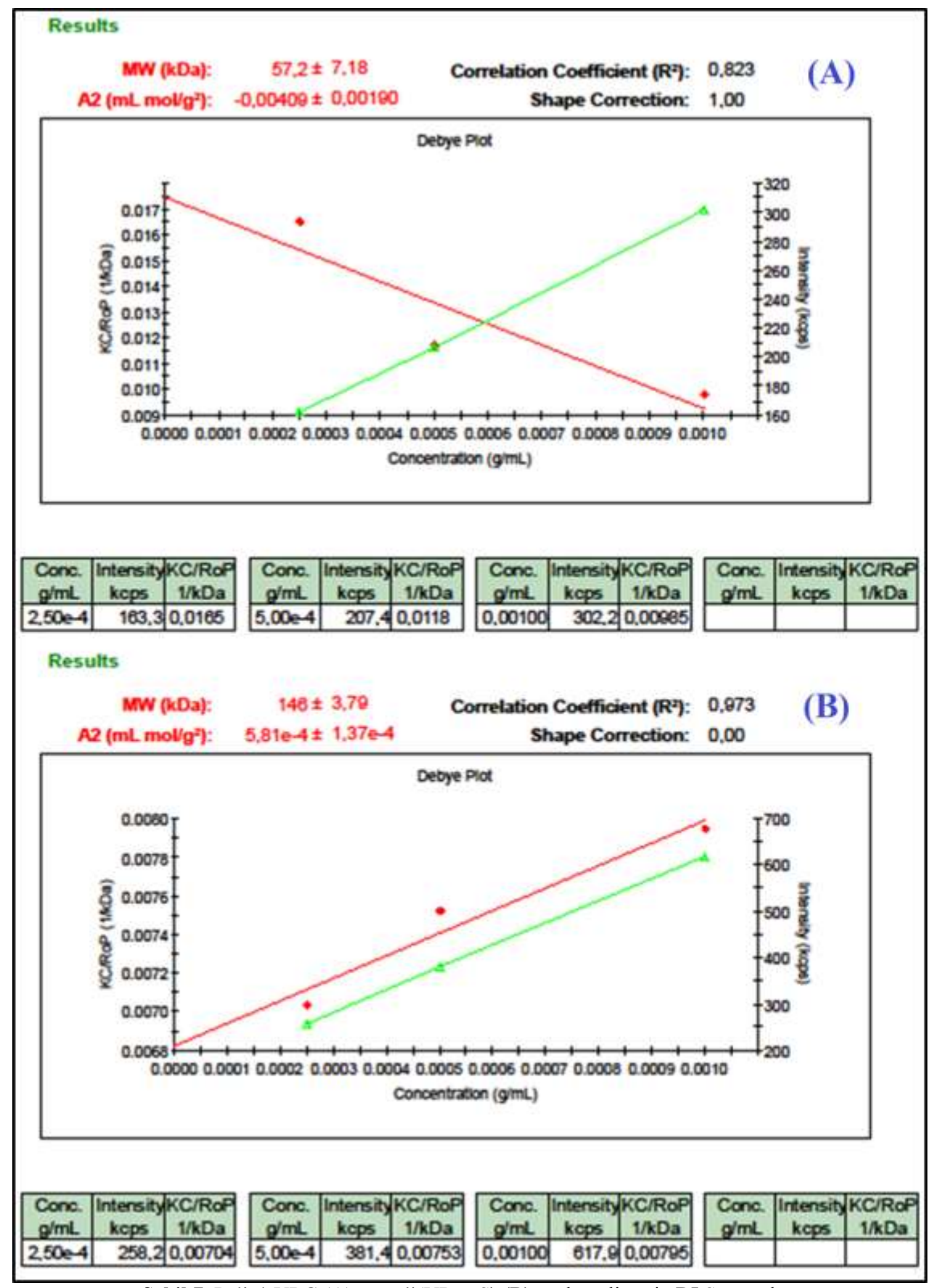

Şekil 7. Poli-4-VBC (A) ve poli(VB-g-S) (B) aşı kopolimerin DLS sonuçları

\section{Sonuç ve Öneriler}

Aşı kopolimer tersinir katılmalı/ayrışmalı zincir transfer (RAFT) ve serbest radikal polimerizasyon (SRP) teknikleri kullanılarak nispeten yüksek verimde ve yüksek moleküler ağırlıkta elde edilmiştir. Bu yöntem aşı kopolimerlerin sentezi için basit ve etkindir. Sentezlenen ürünler ${ }^{1}$ H-NMR, FT-IR, TGA, SEM ve DLS cihazları gibi spektroskopik ve termal analiz teknikleri kullanılarak karakterize edildi. 
Poli(VB-g-S) aşı kopolimerin molekül ağırlığının poli-4-VBC homo polimerinkinden daha büyük olması kopolimerin başarılı bir şekilde sentezlendiğinin kanıtıdır. Çalışmamızda sentezlenen kopolimerin plastik sanayisinde kullanılabileceği düşünülmektedir.

\section{Yazarların Katkısı}

Yazarlar makaleye eşit katkı sağladıklarını beyan ederler.

\section{Çıkar Çatışması Beyanı}

Yazarlar arasında herhangi bir çıkar çatışması bulunmamaktadır.

\section{Araştırma ve Yayın Etiği Beyanı}

Yapılan çalışmada araştırma ve yayın etiğine uyulmuştur.

\section{Kaynaklar}

[1] Ruzette A.V., Leibler L. 2005. Block copolymers in tomorrow's plastics. Nat Mater,4 (1): 19-31.

[2] Öztürk T., Kayğın O., Göktaş M., Hazer B. 2016. Synthesis and Characterization of Graft Copolymers Based on Polyepichlorohydrin via Reversible Addition-Fragmentation Chain Transfer Polymerization. Journal of Macromolecular Science Part A, 53 (6): 362-367.

[3] Bolton J., Rzayev J. 2012. Tandem RAFT-ATRP synthesis of polystyrene-poly(methyl methacrylate) bottle brush block copolymers and their self-assembly into cylindrical nanostructures. ACS Macro Lett, 1 (1): 15-18.

[4] Çatıker E., Öztürk T., Atakay M., Salih B. 2019. Synthesis and characterization of novel aba type poly(ester-ether) triblock copolymers. J Polym Res, 26 (5): 1-9.

[5] Öztürk T., Göktaş M., Savaş B., Işıklar M., Atalar M.N., Hazer B. 2014. Synthesis and characterization of poly(vinyl chloride-graft-2-vinylpyridine) graft copolymers using a novel macroinitiator by reversible addition-fragmentation chain transfer polymerization. E polymers, 14 (1): 27-34.

[6] Altintas O., Tunca U., Barner-Kowollik C. 2011. Star and miktoarm star block (co)polymers via self-assembly of ATRP generated polymer segments featuring Hamilton wedge and cyanuric acid binding motifs. Polym Chem, 2 (5): 1146-1155.

[7] Mo X., Wei F., Tan D., Pang J., Lan C. 2020. The compatibilization of PLA-g-TPU graft copolymer on polylactide/thermoplastic polyurethane blends. J Polym Res., 27 (2): 1-13.

[8] Lin T.A., Lin M.C., Lin J.Y., Lin J.H., Chuang Y.C., Lou C.W. 2020. Modified polypropylene/ thermoplastic polyurethane blends with maleic-anhydride grafted polypropylene: blending morphology and mechanical behaviors. J Polym Res., 27 (2): 1-10.

[9] Erol I., Ozturk A.I. 2005. Free radical copolymerization of novel methacrylates with acrylonitrile and determination of monomer reactivity ratios. J Polym Res., 12 (5): 403-412.

[10] Yasutake M., Hiki S., Andou Y., Nishida H., Endo T. 2003. Physically controlled radical polymerization of vaporized vinyl monomers on surfaces. Synthesis of block copolymers of methyl methacrylate and styrene with a conventional free radical initiator. Macromolecules, 36 (16): 5974-5981.

[11] Simonescu C.I., Comanita E., Pastravanu M., Dimitriu S. 1986. Progress in the field of bi- and poly-functional free-radical polymerization initiators. Prog Polym Sci, 12 (1-2): 1-109.

[12] Tunca U., Karliga B., Ertekin S., Ugur A.L., Sirkecioglu O., Hizal G. 2001. Synthesis of asymmetric difunctional initiators and their use in the preparation of block copolymers via ATRP and SFRP. Polymer, 42 (20): 8489-8493.

[13] Öztürk T., Savaş B., Meyvacı E., Kılıçlıoğlu A., Hazer B. 2020. Synthesis and characterization of the block copolymers using the novel bifunctional initiator by RAFT and FRP technics: evaluation of the primary polymerization parameters. J Polym Res., 27 (3): 1-7.

[14] Hizal G., Sakar D., Tunca U. 2005. Synthesis of tri-arm star diblock co-polymer containing poly(tetrahydrofuran-b-methyl methacrylate) arms via combination of cationic ring-opening 
polymerization and photosensitized free radical polymerization routes. Des Monomers Polym., 8 (6): 609-617.

[15] Chiefari J., Chong Y.K., Ercole F., Krstina J., Jeffery J., Le T.P.T., Mayadunne R.T.A., Meijs G.F., Moad C.L., Moad E., Rizzardo E., Thang S.H. 1998. Living free-radical polymerization by reversible addition-fragmentation chain transfer: the RAFT process. Macromolecules, 31 (16): 5559-5562.

[16] Kawahara N., Kojoh S., Matsuo S., Kaneko H., Matsugi T., Saito J., Kashiwa N. 2006. Synthetic method of polyethylenepoly(methylmethacrylate) (PE-PMMA) polymer hybrid via reversible addition-fragmentation chain transfer (RAFT) polymerization with functionalized polyethylene. Polym Bull, 57 (6): 805-812.

[17] Goto A., Tsujii Y., Fukuda T. 2008. Reversible chain transfer catalyzed polymerization (RTCP): a new class of living radical polymerization. Polymer, 49 (24): 5177-5185.

[18] Mitsoni E., Roka N., Pitsikalis M. 2019. Statistical copolymerization of N-vinyl-pyrrolidone and alkyl methacrylates via RAFT: reactivity ratios and thermal analysis. J Polym Res., 26 (5): 1-12.

[19] Hazer B., Arslan H., Senemoğlu Y., Şen Ş. 2019. Synthesis of block/ graft copolymers based on vinyl benzyl chloride via reversible addition fragmentation chain transfer (raft) polymerization using the carboxylic acid functionalized trithiocarbonate. J Polym Res., 26 (5): 1-19.

[20] Öztürk T., Atalar M.N., Göktaş M., Hazer B. 2013. One-step synthesis of block-graft copolymers via simultaneous reversible-addition fragmentation chain transfer and ring-opening polymerization using a novel macroinitiator. J Polym Sci A Polym Chem, 51 (12): 2651-2659.

[21] Öztürk T., Hazer B. 2010. Synthesis and characterization of a novel macromonomer initiator for reversible addition fragmentation chain transfer (RAFT). Evaluation of the polymerization kinetics and gelation behaviors. J Macromol Sci Part A Pure Appl Chem, 47 (3):2 65-272.

[22] Şanal T., Oruç O., Öztürk T., Hazer B. 2015. Synthesis of pH- and thermo-responsive poly( $\varepsilon-$ caprolactone-b-4-vinyl benzyl-g-dimethyl amino ethyl methacrylate) brush type graft copolymers via RAFT polymerization. J Polym Res., 22 (2): 1-12.

[23] Montoya-Villegas K.A., Licea-Claveríe Á., Zapata-González I., Gómez E., Ramírez-Jiménez A. 2019. "The effect in the RAFT polymerization of two oligo(ethylene glycol) methacrylates when the CTA 4-cyano-4-(propylthiocarbonothioylthio) pentanoic acid is auto-hydrolyzed to its corresponding amide. J Polym Res., 26 (3): 1-11.

[24] Liu C., Ren X. 2018. Synthesis and characterization of controlled benzotriazole light stabilizer by RAFT polymerization and its application. J Polym Res., 25 (4): 1-12.

[25] Liu S., Tian L., Mao H., Ning W., Shang P., Wu J., Shi X. 2018. Micellization and sol-gel transition of novel thermo- and pH-responsive ABC triblock copolymer synthesized by RAFT. J Polym Res., 25 (12): 1-12.

[26] Braun D. 1970. Polistirenin kimyasal değişiklikleri. Kunststoffe, 50: 375.

[27] Kurbanova R., Okudan A., Mirzaoglu R., Kurbanov S., Karatas İ., Ersöz M., Özcan E., Ahmedova G., Pamuk V. 1998. Effects of the functionals groups of polystyrene on its adhesion improvoment and corrsion resistance. J. Adh. Sci. Techn., 12 (9): 947-955. 